\title{
Nivel de conocimiento de bioseguridad en trabajadores administrativos, Hospital Regional Virgen de Fátima, Chachapoyas, 2019
}

\author{
Story workshop mother nature to improve the environmental attitudes of primary \\ students, Luya 2018 \\ Mabel Yesenia Cruz Ramos ${ }^{1}$, Carla María Ordinola Ramírez ${ }^{2}$
}

\section{RESUMEN}

El objetivo del presente estudio fue determinar el nivel de conocimiento de bioseguridad en trabajadores administrativos, Hospital Regional Virgen de Fátima, Chachapoyas, 2019. Se aplicó el enfoque cuantitativo, nivel descriptivo, de tipo observacional, prospectivo, transversal y de análisis estadístico univariado. La población estuvo constituido por todos los trabajadores administrativos en un total de 85 , se trabajó con el $100 \%$ de la población. Los datos se recolectaron mediante el cuestionario de conocimiento validado con un nivel de significancia $\alpha=0.05$ ( $95 \%$ de nivel de confianza y un $5 \%$ de margen de error). Los resultados indican que el $57.6 \%$ de los trabajadores administrativos presentaron un nivel de conocimiento regular, el $37.6 \%$ bueno y el $4.7 \%$ bajo. Se concluye que más de la mitad de los trabajadores administrativos del Hospital Regional Virgen de Fátima tienen un conocimiento en el nivel de regular a bueno en lo que respecta a medidas de bioseguridad y en la dimensión del uso de ambiente y la dimensión de uso personal, la mitad de los trabajadores administrativos tienen un conocimiento bueno seguida de un conocimiento regular, mientras que en el uso de equipos tienen un conocimiento entre regular y bueno de forma proporcional sobre medidas de bioseguridad.

Palabras clave: Actitud ambiental, taller de cuentos ambientales.

\section{ABSTRACT}

The objective of this study was to determine the level of knowledge of biosafety in administrative workers, Hospital Regional Virgen de Fátima, Chachapoyas, 2019. The quantitative approach, descriptive level, observational, prospective, cross-sectional and univariate statistical analysis was applied. The population consisted of all the administrative workers in a total of 85 , working with $100 \%$ of the population. Data were collected through the validated knowledge questionnaire with a significance level $\alpha=0.05$ (95\% confidence level and $5 \%$ margin of error). The results indicate that $57.6 \%$ of the administrative workers presented a regular level of knowledge, 37.6\% good and 4.7\% low. It is concluded that more than half of the administrative workers of the Virgen de Fátima Regional Hospital have knowledge at the level from fair to good with regard to biosafety measures and in the dimension of the use of the environment and the dimension of personal use, half of the administrative workers have a good knowledge followed by a regular knowledge, while in the use of equipment they have a knowledge between fair and good proportionally on biosecurity measures.

Keywords: Knowledge, biosecurity, administrative workers.

\footnotetext{
Bach. Tecnología Médica - Terapia Física y Rehabilitación, Universidad Nacional Toribio Rodríguez de Mendoza de Amazonas, mabe_cr@hotmail.com

${ }^{2}$ Dr. $\overline{M g}$. Carla María Ordinola Ramírez docente auxiliar a dedicación exclusiva, Universidad Nacional Toribio Rodríguez de Mendoza de Amazonas, carla.ordinola@untrm.edu.pe
} 


\section{INTRODUCCIÓN}

La bioseguridad debe entenderse como una doctrina de comportamiento encaminada a lograr actitudes y conductas que disminuyan el riesgo del trabajador de la salud de adquirir infecciones en el medio laboral. (Sánchez, 2015, p. 29)

Según la organización mundial de la salud (OMS), la bioseguridad tiene por objeto prevenir la exposición involuntaria a agentes patógenos o la liberación accidental de estos. Considerar el tema de bioseguridad para un centro de salud no es solamente tener contratada a una empresa para que retire los desechos biológicos y usar guantes, es algo mucho más integral que tiene que ver no solo con la salud del personal involucrado sino con toda la sociedad. (Arrizabalaga, 2013, p. 25).

La Organización Internacional del Trabajo (OIT) y la Organización Panamericana de la Salud (OPS) estiman que cada año se producen 250 millones de accidentes laborales en todo el mundo y 3000 personas mueren cada día por causas relacionadas con el trabajo. Así, las causas de muerte vinculadas al trabajo se colocan por encima de los accidentes de tránsito, las guerras y la violencia.

Pese a que año tras año trabajan con las normas de bioseguridad y se implementan directivas para evitar accidentes laborales se observa que sólo en el año 2011 el Hospital Guillermo Almenara registró 304 accidentes de trabajo de los cuales 116 fueron punzocortantes. Si bien en el Perú, el 50\% de los accidentes punzocortantes no se reportan debido a la falta de cultura de seguridad en salud, las estadísticas señalan que las lesiones por pinchazos se dan mayormente por agujas de aspiración (27\%), agujas para suturas (19\%) y bisturís (7\%). Asimismo, se registra que el $44 \%$ de lesiones por agujas y otros instrumentos punzocortantes se generaron en enfermería y el $27 \%$ en médicos. (Alarcón \& Rubiños, 2015, p. 22).

Con toda la problemática descrita se formuló la siguiente interrogante: ¿Cuál es el nivel de conocimiento de bioseguridad en trabajadores administrativos, Hospital Regional Virgen de Fátima, Chachapoyas - 2019?, cuyo objetivo general fue: Determinar el nivel de conocimiento de bioseguridad en trabajadores administrativos, Hospital Regional Virgen de Fátima, Chachapoyas 2019. Objetivos específicos: identificar el nivel de conocimiento de bioseguridad en el uso del ambiente, uso de equipos, para el personal, en trabajadores administrativos, Hospital Regional Virgen de Fátima, Chachapoyas - 2019.
La importancia de este estudio fue dar a conocer el nivel de conocimiento de bioseguridad en los trabajadores administrativos, Así mismo los resultados del presente estudio servirán como un precedente en la región, ya que en la actualidad no se cuenta con trabajos relacionados al tema; aunque una de sus limitantes es el hecho que servirá solo para la población en estudio, pudiendo ser tomada como referente en futuras investigaciones.

\section{MATERIALES Y MÉTODOS}

\section{Diseño de investigación}

Se utilizó el diseño descriptivo, cuyo diagrama será el siguiente:

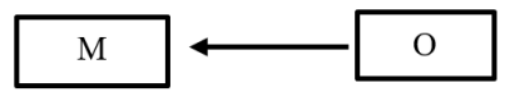

Donde:

$\mathrm{M}=$ Muestra de estudio.

$\mathrm{O}=$ Nivel de conocimiento sobre las normas de bioseguridad

\subsection{Población, muestra y muestreo}

\section{Población:}

Estuvo constituido por todos los trabajadores administrativos, en total de 85 trabajadores.

\section{Criterios de inclusión}

-Trabajadores administrativos de ambos sexos.

- Trabajadores administrativos nombrados, contratados y por locación.

\section{Criterios de exclusión}

-Trabajadores administrativos de ambos sexos que no deseen participar de la investigación.

\section{Muestra:}

En este estudio no se utilizó ningún tipo de muestreo, ya que la muestra fue considerada al $100 \%$ de la población y este el 100\% del universo.

\subsection{Métodos, técnicas e instrumentos de recolección de datos:}

Se utilizó un cuestionario de conocimiento con 15 preguntas. La variable se midió con la escala de Razón. En tres categorías: alto, medio y bajo. Cuyos puntajes fueron:

0 - 5 puntos: Nivel de conocimiento malo.

06 -10 puntos: Nivel de conocimiento regular.

11 -15 puntos: Nivel de conocimiento bueno. 


\section{Validación y confiabilidad}

El instrumento se encuentra validado por Robles, K. (2017). En su estudio "Nivel de conocimiento y prácticas de bioseguridad del personal de salud en los servicios de Cirugía del Hospital Nacional Dos de Mayo 2017". Validó el instrumento con un nivel de significancia alfa $=0.05$ y un intervalo de confianza de $95 \%$ y $5 \%$ de probabilidad de error.

\subsection{Análisis de datos}

La información final se procesó en el paquete estadístico SPSS Statistics 23; las hipótesis se contrastaron mediante la prueba estadística paramétrica de la $\mathrm{t}$ - student con un nivel de significancia del 0.05 (5\% de margen de error y el $95 \%$ de confiabilidad). Los resultados se presentaron en tablas y figuras.

\section{RESULTADOS}

Tabla 1. Nivel de conocimiento de bioseguridad, en trabajadores administrativos, Hospital Regional Virgen de Fátima, Chachapoyas, 2019.

\begin{tabular}{ccc}
\hline $\begin{array}{c}\text { NIVEL DE } \\
\text { CONOCIMIENTO }\end{array}$ & fi & \% \\
\hline Malo & 4 & 4.7 \\
Regular & 49 & 57.6 \\
Bueno & 32 & 37.6 \\
\hline TOTAL & $\mathbf{8 5}$ & $\mathbf{1 0 0 . 0}$ \\
\hline
\end{tabular}

Tabla 2. Nivel de conocimiento general de bioseguridad según dimensiones, en trabajadores administrativos, Hospital Regional Virgen de Fátima, Chachapoyas, 2019.

\begin{tabular}{|c|c|c|c|c|c|c|}
\hline \multirow{3}{*}{$\begin{array}{l}\text { Nivel de } \\
\text { conocimie } \\
\text { nto } \\
\text { general }\end{array}$} & \multicolumn{6}{|c|}{ Dimensiones de la bioseguridad } \\
\hline & \multicolumn{2}{|c|}{$\begin{array}{l}\text { Uso de } \\
\text { ambiente }\end{array}$} & \multicolumn{2}{|c|}{$\begin{array}{l}\text { Uso de } \\
\text { equipos }\end{array}$} & \multicolumn{2}{|c|}{$\begin{array}{l}\text { Uso } \\
\text { personal }\end{array}$} \\
\hline & fi & $\%$ & fi & $\%$ & fi & $\%$ \\
\hline Malo & 10 & 11.8 & 12 & 14.1 & 14 & 16.5 \\
\hline Regular & 32 & 37.6 & 37 & 43.5 & 28 & 32.9 \\
\hline Bueno & 43 & 50.6 & 36 & 42.4 & 43 & 50.6 \\
\hline $81 \%$ & 05 & 100.0 & 85 & 100.0 & 85 & 100.0 \\
\hline
\end{tabular}

\section{DISCUSIÓN}

De acuerdo a los resultados encontrados se determinó que el $57.6 \%$ de los trabajadores administrativos presentó un nivel de conocimiento regular, quiere decir que los trabajadores conocen parcialmente las normas de bioseguridad en función al uso de ambiente, al uso de equipos y al uso personal, el $37.6 \%$ bueno, y el $4.7 \%$ malo. (Tabla 1 ).

Además se observa que en los trabajadores administrativos encuestados sobre las normas de bioseguridad según dimensiones: uso de ambiente, el $50.6 \%$ presentaron un nivel de conocimiento bueno, el $37.6 \%$ regular, y el $11.8 \%$ malo. Uso de equipos, el $42.4 \%$ presentaron un nivel de conocimiento bueno, el $43.5 \%$ regular, y el $14.1 \%$ malo. Uso personal, el $50.6 \%$ presentaron un nivel de conocimiento bueno, el $32.9 \%$ regular, y el $16.5 \%$ malo. (Tabla 2 )

Los resultados del presente estudio es muy diferente a los resultados de López, D. (2012); Robles, K. (2017); Cama, L. (2014); Arrizabalaga, J. (2013) y García, F. (2012); ya que en estos estudios se determinó un nivel de conocimiento alto sobre las normas de bioseguridad, principalmente a su experiencia ganada desempeñando sus labores y a la formación recibida en su casa de estudios.

Como se puede apreciar los resultados son muy diferentes, en la cual en el presente estudio prevalece un nivel de conocimiento regular mientras que en los demás estudios un nivel de conocimiento alto, esta diferencia se puede deber a muchos factores como diferentes poblaciones con realidades diferentes, entre otros.

Las normas de bioseguridad son medidas preventivas que se implementan en las instituciones para proteger la salud y disminuir el riesgo de transmisión de microorganismos, tos cuales están presentes en el ambiente, especialmente en el área hospitalaria. Así mismo el elemento más importante de la bioseguridad es el estricto cumplimiento de las prácticas y procedimientos apropiados y el uso eficiente de materiales y equipos, los cuates constituyen la primera barrera a nivel de contención para el personal y el medio. (García, 2011, p. 27).

En la actualidad existe un renovado sentido de vigilancia acerca de lo que el personal de un Hospital debe conocer y practicar para protegerse y de este modo minimizar o evitar los riesgos de contaminación en el lugar donde se desempeña. Siendo por ende primordial que el profesional conozca y utilice de manera adecuada las normas de bioseguridad, a fin de resguardar su integridad física y proteger de igual manera a los pacientes que atiende. Cumpliendo con el principio de universalidad, a través del cual se establece el deber de involucrar a todos los pacientes de todos los servicios, independientemente de conocer o no su serología en la aplicación y conocimiento de las medidas de Bioseguridad. La importancia del estudio radicara 
en dar a conocer el nivel de conocimiento de bioseguridad en los trabajadores administrativos.

\section{CONCLUSIONES}

1. Más de la mitad de los trabajadores administrativos del Hospital Regional Virgen de Fátima tienen un conocimiento en el nivel de regular a bueno en lo que respecta a medidas de bioseguridad.

2. En la dimensión del uso de ambiente y la dimensión de uso personal la mitad de los trabajadores administrativos tienen un conocimiento bueno seguida de un conocimiento regular, mientras que en el uso de equipos tienen un conocimiento entre regular y bueno de forma proporcional sobre medidas de bioseguridad.

\section{REFERENCIAS BIBLIOGRÁFICAS}

Alarcón, M. \& Rubiños, D. (2015). Conocimientos y prácticas en la prevención de riesgos biológicos de los profesionales de salud del Hospital Belén Lambayeque. Disponible en: http://publicaciones.usat.edu.pe/index.php/ AccCietna2014/article/view/331

Arrizabalaga, J. (2013). Medidas de Bioseguridad que aplica el profesional Tecnólogo Médico en el Hospital Universitario San Vicente de Paúl. Tesis para optar el título de Licenciatura. Universidad de Sao Paulo. Brasil

Cama, L. (2014). Relación entre conocimientos y prácticas de las medidas de bioseguridad por contacto con fluidos corporales que realiza la enfermera(o) del Servicio de Emergencia, Hospital Nacional Dos de Mayo. Lima. (Tesis para optar el título de Licenciada en Enfermería). Facultad de Medicina Humana. E.P. de Enfermería. Universidad Nacional Mayor de San Marcos.

García, F. (2012). Relación entre los conocimientos de las medidas de Protección Ocupacional que tiene el Tecnólogo Médico y aplicación en la atención del paciente con SIDA, en los Servicios de Medicina del HNGAI. Perú. Tesis para optar Licenciatura en Enfermería. Facultad de Enfermería. UNMSM.

López, D. (2012). "Nivel de conocimientos y prácticas de medidas de bioseguridad en trabajadores del servicio de Terapia Físicay rehabilitación del Hospital MINSA 11-2 Tarapoto junio - agosto 2012".

Robles, K. (2017). Nivel de conocimiento y prácticas de bioseguridad del personal de salud en los servicios de Cirugía del Hospital Nacional Dos de Mayo 2017. Disponible en: http://repositorio.ucv.edu.pe/bitstream/hand le/UCV/7037/Robles_OKM.pdf?sequence= $1 \&$ isAllowed $=\mathrm{y}$

Sánchez, A. (2015). Manual de bioseguridad para el diplomado en enfermería. Editorial MAD. $S . L$, primera edición. Obtenido: books.google.com.ec/books? isbn $=8466522751$. 